\title{
Advanced fit technique for astrophysical spectra
}

\section{Approach insensitive to a large fraction of outliers}

\author{
S. Bukvić ${ }^{1}$, Dj. Spasojević ${ }^{1}$, and V. Žigman ${ }^{2}$ \\ 1 Faculty of Physics, University of Belgrade, Studentski Trg 12-16, Belgrade, Serbia \\ e-mail: ebukvic@ff.bg.ac.yu \\ 2 University of Nova Gorica, Vipavska cesta 13, Nova Gorica, Slovenia
}

Received 4 July 2006 / Accepted 23 September 2007

\section{ABSTRACT}

\begin{abstract}
Aims. The purpose of this paper is to introduce a robust method of data fitting convenient for dealing with astrophysical spectra contaminated by a large fraction of outliers.

Methods. We base our approach on the suitable defined measure: the density of the least squares (DLS) that characterizes subsets of the whole data set. The best-fit parameters are obtained by the least-square method on a subset having the maximum value of DLS or, less formally, on the largest subset free of outliers.

Results. We give the FORTRAN90 source code ${ }^{\star}$ of the subroutine that implements the DLS method. The efficiency of the DLS method is demonstrated on a few examples: estimation of continuum in the presence of spectral lines, estimation of spectral line parameters in the presence of outliers, and estimation of the thermodynamic temperature from the spectrum that is rich in spectral lines.

Conclusions. Comparison of the present results with the ones obtained with the widely used comprehensive multi-component fit yields agreement within error margins. Due to simplicity and robustness, the proposed approach could be the method of choice whenever outliers are present, or whenever unwelcome features of the spectrum are to be considered as formal outliers (e.g. spectral lines while estimating continuum).
\end{abstract}

Key words. methods: data analysis - methods: numerical - techniques: spectroscopic - line: profiles

\section{Introduction}

The task often met in data processing is to find the best parameters of some model function that would represent a particular set of data in the best-fit sense. For this purpose it is a common practice to use the least squares as a merit function for various data fittings. Unfortunately, measurements are not free of errors, which occasionally cause some experimental points to simply be way off. Also, data of interest are frequently spoiled by the irregular deviation of a certain number of data points due to some undesirable process that cannot be avoided. This can easily turn the results obtained with the least-square method into nonsense.

The reliability of the best-fit could be significantly enhanced if outliers could be successfully recognized and subsequently removed. A variety of statistical tests, reviewed in (Barnett \& Lewis 1994), have been developed to decide whether a data point is an outlier or not. If there is a sufficient number of replicate points at each value of $x$, these tests could be useful for determining whether a single value is a significant outlier. Unfortunately, each point is measured only once in typical experiments. In such a case, one can choose to perform an outlier test on the entire set of residuals of the least-square fit. The problem arises if outliers are allocated asymmetrically. In this case the best-fit curve tends to be inclined towards the outliers, so that outlying points might not be recognized.

* A full-code demo program is available in electronic form at the CDS via anonymous ftp to

cdsarc.u-strasbg.fr $(130.79 .128 .5)$ or via

http://cdsweb.u-strasbg.fr/cgi-bin/qcat?]/A+A/477/967
In order to minimize the impact of outliers, a multitude of robust approaches has been proposed (Hampel et al. 1986; Hoaglin et al. 1983). While efficient in the case of symmetrically allocated outliers, most of the robust fit approaches are scaledependent and ineffective if a large fraction of outlying points lie on the same side of the best-fit curve. Some relevant ideas for how to deal with outliers in case of a linear, as well as nonlinear, fit are presented in (Marx 1996; Motulsky \& Brown 2006; Liu et al. 2005).

Well-known tasks to which the least-square method is not directly applicable include determining the spectrum base line and spectrum continuum. Both issues often arise in spectroscopy, since for many applications in astrophysics (Contini \& Viegas 2000; Rossa et al. 2000; Peterson et al. 2002; Freudling et al. 2003; Gilbert \& Peterson 2003; Gabel et al. 2005) and in physics (Meisel \& Cote 1992; Spasojević et al. 1996), accurate knowledge of both the local continuum value and signal base line is essential. To overcome the drawbacks of the least squares, a few specific, non robust approaches have been proposed. One of them is to estimate the continuum and power-law exponents from the spectral regions uncontaminated by emission or absorption lines (Gabel et al. 2005; Liu \& Yan 2005; Wang et al. 2005). Within this approach, the spectral lines are unwelcome features formal outliers, which can produce spurious results. Another approach is to apply a complex multi-component fit (Dietrich et al. 2002; Freudling et al. 2003; Gabel et al. 2005; Dietrich et al. 2005), which takes all known features of the spectrum into account. However, some unknown, non-implied features, or true 
outlying points can still induce inaccurate estimates of the fit coefficients.

In a recent paper (Bukvić \& Spasojević 2005), a robust method for estimating of the spectrum base line and continuum from large data sets has been proposed. The method was based on the close points concept (CPC), capable to distinguish outliers from close points, with the resulting best-fit parameters obtained only on the set of close points.

In this paper we develop this technique further by applying the least-square approach in accordance with CPC. The proposed method is insensitive to the presence of a large number of outlying points, which makes it appropriate for various purposes. For example, in spectrum analysis, it can be used to fit the full spectrum (base line/continuum + all spectral lines), to fit only selected spectrum characteristics (base line/continuum + selected spectral lines, while all other spectral lines are treated as outliers), or to fit the base line/continuum only (with all spectral lines being treated as outliers).

\section{Merit function}

Consider a data set $s_{0}=\left\{\left(x_{i}, y_{i}\right): i=1,2, \ldots, n_{0}\right\}$ consisting of $n_{0}$ experimental points and a family of model functions $f\left(x ; a_{1}, a_{2}, \ldots, a_{m}\right)$ depending on $x$ and on the parameters $a_{1}, \ldots, a_{m} \equiv \mathbf{a}$, specifying a model function from the family. In the least-square approach, the best model function is usually found by applying the method to the whole data set $s_{0}$. However, the set $s_{0}$ may be contaminated by various types of unwelcome data. Therefore, it could happen that the model function that optimally reflects the features of interest actually corresponds not to the initial data set $s_{0}$, but rather to some of its subsets, $s_{b}-$ the "best" one - not known in advance.

In order to identify it, we introduce the density of least squares - the quantity suitable for characterizing the initial data set $s_{0}$ and any particular subset $s$, in the following way:

$D_{k}(s)=\frac{\sum_{s} d_{i}^{2}}{d_{\max }^{k}(s)}$.

Here, symbol $\Sigma_{s}$ denotes summation over all data points of subset $s$, and $d_{i}=\left|y_{i}-f\left(x_{i} ; \mathbf{a}(s)\right)\right|$ is the absolute value of the deviation of $y_{i}$ from the model function $f\left(x_{i} ; \mathbf{a}(s)\right)$, defined here as distance of data point $\left(x_{i}, y_{i}\right) \in s$ from $f\left(x_{i} ; \mathbf{a}(s)\right)$, as specified by $\mathbf{a}(s)$. The parameters $\mathbf{a}(s)$ are the best-fit parameters, obtained by applying the ordinary least-square (OLS) method to the subset $s$, so that $\sum d_{i}^{2}$ is the corresponding OLS sum for that subset. Analogously, $d_{\max }(s)=\max \left\{d_{i}\right\}$ is the maximal distance, which specifies the width of subset $s$, relative to the model function $f\left(x_{i} ; \mathbf{a}(s)\right)$. The exponent $k$ is a real number, whose value is yet to be determined. It will be shown in Sect. 3 that $2 \leq k<3$, and that the choice $k=2$ additionally simplifies our method.

The subset $s_{b}$, singled out by having maximum density of the least squares, is taken as the best subset; its OLS best-fit parameters $\mathbf{a}\left(s_{b}\right)$ are considered to yield the most reliable estimate of the features of interest, hence, are accepted as the final outcome of the proposed method. The estimation of uncertainties $\Delta \mathbf{a}\left(s_{b}\right)$ of the OLS best-fit parameters $\mathbf{a}\left(s_{b}\right)$ is explained in Sect. 4 . To conform to standard terminology, we introduce the merit function

$\mu_{k}(s)=-D_{k}(s)$,

which is a function of subset $s$ of the data set $s_{0}$. Obviously, on subset $s_{b}$, merit function (2) attains its minimum value, whereas $D_{k}(s)$ attains its maximum value.
We call the foregoing approach the density of least-square (DLS) method. We call the points from subset $s_{b}$ close points, while all other points we call distant points or outliers with respect to the model function $f\left(x_{i} ; \mathbf{a}(s)\right)$. In the same sense, the width of the best subset $d_{b}=d_{\max }\left(s_{b}\right)$ can be conveniently used to set a discrimination level between distant and close points.

So far, we have considered data sets with unspecified errors of ordinates $y_{i},\left(i=1,2, \ldots, n_{0}\right)$. If individual errors $\sigma_{i}$ are, on the other hand, assigned to corresponding $y_{i}$ then, instead of geometrical distances $d_{i}$, relative distances $\delta_{i}=d_{i} / \sigma_{i}$ should be used. Accordingly, the density of weighted least squares is defined by an appropriate extension of Eq. (1):

$D_{\mathrm{W}, k}(s)=\frac{\sum_{s} \delta_{i}^{2}}{\delta_{\max }^{k}(s)}$.

The geometrical interpretation of Eq. (3) is, however, less obvious. In what follows we will, therefore, refer mostly to Eq. (1).

As shown in the Appendix, the best-fit parameters $\mathbf{a}\left(s_{b}\right)$ found by the DLS method are, actually, the maximum likelihood parameters, obtained by a specific Local maximum-likelihood estimator. This evidence, together with the calibration procedure put forward in Sect. 3.1, forms the formal basis of the DLS method.

Now we proceed to intuitive considerations, illustrating how DLS works in the case $k=2$. Let $s_{0}$ be a data set containing one outlying point with respect to a model function, and let $s$ be its subset that contains all points from $s_{0}$, except the outlying point. Although both the numerator $\sum_{s} d_{i}^{2}$ and denominator $d_{\max }^{2}(s)$ in Eq. (1) are smaller on $s$ than on $s_{0}$, the effect of the decrease in $d_{\max }(s)$ in $\sum_{s} d_{i}^{2}$ is lessened by the contribution of other points, so that the denominator decrease becomes dominant. It follows that the density $D_{k}(s)$ of the subset $s$ will typically be higher than $D_{k}\left(s_{0}\right)$. More generally, if the data set $s_{0}$ contains outlying points, then the density of least squares typically attains its maximum value on some subset that is free of outliers, but still contains a large percentage of data.

With respect to definition (2), we would like to emphasize that we do not simply seek for parameters that minimize the value of merit function $\mu$ on the initial data set. Instead, we are presently concerned with finding the best subset $s_{b}$ for which the density of least squares exhibits its maximum. This task cannot be reduced to the standard minimization problem.

Indeed, the standard minimization problem takes place in a multidimensional space of model function parameters. Provided the initial guess is given, the standard minimization algorithms follow the gradient direction, assuring rapid approach to some local minimum. For functions that, like merit function (2), have data subset as an argument some appropriate substitute for the gradient minimization algorithm is to be sought; see the discussion for details. To this end we establish an ordered collection of data subsets in agreement with our main intention of removing outlying points, so as to obtain the subset with the maximum density of least squares.

We define the ordered collection of data subsets by iterating the following two-stage procedure. We first calculate the OLS best-fit model function for the given subset $s$ and then remove from $s$ the point with the greatest distance $d=d_{\max }(s)$ with respect to the best-fit curve. In the second stage, we apply OLS only to the retained data points and recalculate distances $d_{i}$ of the remaining data points from the new best-fit model function. If there are points with distances larger than the starting $d_{\max }(s)$, we remove them as well. We repeat these steps until all points with distances $d_{i} \geq d_{\max }(s)$ are removed. The smaller subset of 


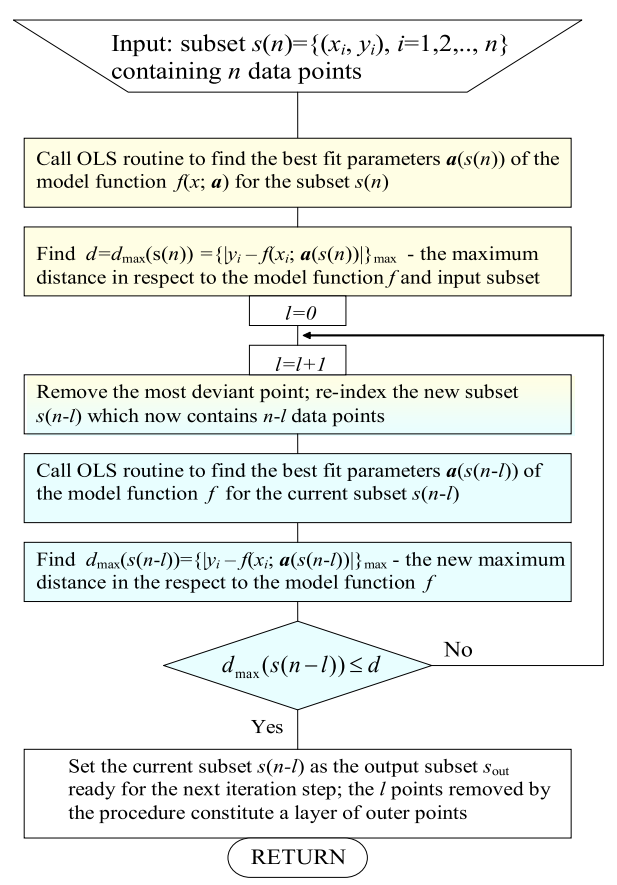

Fig. 1. Flowchart of the procedure used to obtain the ordered collection $C$. Index $j=n-l$ denotes the number of points in the subset $s(j)$. Yellow and blue colors indicate algorithm steps of the first and of the second stages, respectively. The algorithm step painted in both colors is invoked only once within the first stage, at the very beginning; all its subsequent calls are within the second stage.

retained points, with new $d_{\max }$, is now ready for the next iteration cycle. Points eliminated from $s$ by this procedure, see Fig. 1, constitute a layer of outer points for subset $s$ or, simply, layer ${ }^{1}$.

By using the foregoing procedure, we iteratively remove layer-by-layer from the initial data set $s_{0}$, and we eventually

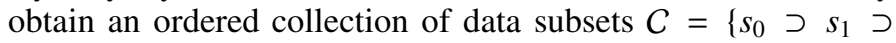
$\left.s_{2}, \ldots, \supset s_{p} \ldots\right\}$ where index $p$ counts the subsets in the collection. After each iteration, we calculate $D_{k}\left(s_{p}\right)$. Although one can fit the subset of $n$ data points to a $m$-parameter model function technically whenever $n \geq m$, we terminate the build-up of collection $C$ whenever a more conservative condition $n<m+3$ is met, or after $D_{k}\left(s_{p}\right)$ becomes indefinite - see Sect. 3.2. As the best subset $s_{b}$, we accept the subset from collection $C$ having a maximum value of DLS. For the users' convenience, a FORTRAN code of the DLS data-fitting method is given in Sect. 8.2.

\section{Calibration procedure}

\subsection{Normal distribution case}

Let the data set $s_{0}=\left\{\left(x_{i}, y_{i}\right): i=1,2, \ldots, n_{0}\right\}$ contain $n_{0}$ normally distributed points around the value $y=0$. We assume that the number $n_{0}$ is large enough for the statistical parameters related to $s_{0}$ to be indistinguishable from their corresponding limits, when $n_{0} \rightarrow \infty$.

${ }^{1}$ In applications the coarsened version of this rule is of particular interest. The difference is only in the first stage of this procedure: instead of removing a point with $d_{i}=d_{\max }$, we remove all points such that their distances $d_{i} \geq r d_{\max }(s)$, where $0<r \leq 1$ is a removal parameter. For $r=1$ we are back at the original rule, while for $r<1$ we remove more points in a single step.

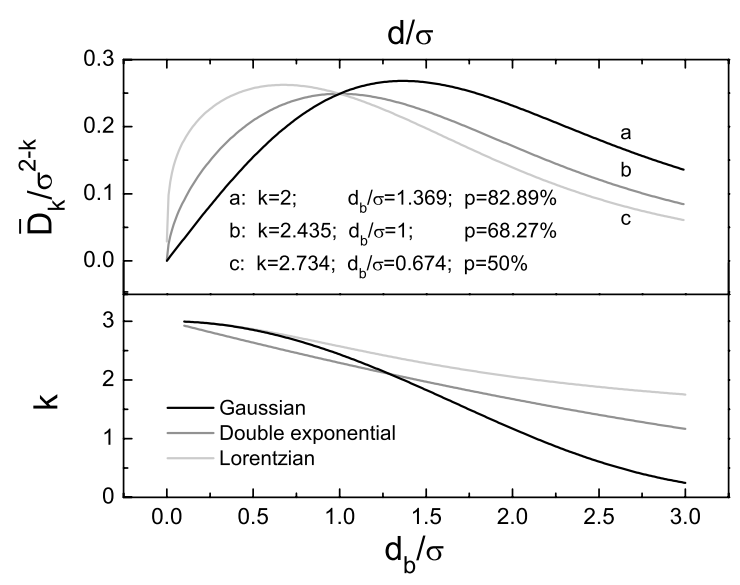

Fig. 2. Top panel: $\bar{D}_{k} / \sigma^{2-k}$ versus relative width $d / \sigma$ of $d$-stripes for Gaussian distribution. Presented curves correspond to three characteristic values of $k$, as given in the legend, together with abscissae $d_{b} / \sigma$ of the corresponding maxima; $p$ is the percentage of points contained in the corresponding $d_{b}$-stripes. Bottom panel: exponent $k$ versus $d_{b} / \sigma$, according to Eq. (6) for Gaussian distribution - bold curve. As indicated, analogous curves for Lorentzian and for double-exponential distribution are also given. In DLS method we only use the values $2 \leq k<3$.

Then, the number $\mathrm{d} n$ of $y_{i}$ 's in the interval $(y-\mathrm{d} y / 2, y+$ $\mathrm{d} y / 2)$ is

$\mathrm{d} n(y) \approx \frac{n_{0}}{\sigma \sqrt{2 \pi}} \cdot \exp \left(-\frac{y^{2}}{2 \sigma^{2}}\right) \mathrm{d} y$,

where $\sigma$ is the corresponding standard deviation. In this particular case, we take the horizontal lines $y=$ const. for the family of model functions. Let $s_{d}$ be a $d$-stripe, i.e. the subset consisting of all points from $s_{0}$ located between the two horizontal lines: $y=-d$ and $y=d$. For $s_{d}$ we see that $\sum d_{i}^{2} \approx \int_{-d}^{+d} y^{2} \mathrm{~d} n(y)$, so that $D_{k}\left(s_{d}\right) / n_{0} \approx \bar{D}_{k}(d)$, where

$$
\begin{aligned}
\bar{D}_{k}(d) & =\frac{1}{d^{k} \sigma \sqrt{2 \pi}} \int_{-d}^{+d} y^{2} \exp \left(-\frac{y^{2}}{2 \sigma^{2}}\right) \mathrm{d} y \\
& =\frac{\sigma^{2-k}(d / \sigma)^{-k}}{\sqrt{2 \pi}} \int_{-(d / \sigma)}^{+(d / \sigma)} t^{2} \exp \left(-t^{2} / 2\right) \mathrm{d} t
\end{aligned}
$$

is the DLS per one data point for $d$-stripes, in the case of Gaussian distribution.

On the other hand, one can show that inequality $n_{0} \bar{D}_{k}(d) \geq$ $D_{k}(s)$ holds $^{2}$ for any subset $s$ with $d_{\max }(s)=d$, implying that the maximum of $n_{0} \bar{D}_{k}(d)$ is equal to the DLS maximum ${ }^{3}$. The same maximum is found in the ordered collection $C$, since $C$ consists of $d$-stripes. In the top panel of Fig. 2 we present plots of $\bar{D}_{k}(d)$ scaled by $\sigma^{2-k}$ for three characteristic values of parameter $k$; each curve manifests distinct maximum.

\footnotetext{
${ }^{2}$ More precisely, for subsets $s$ such that $d_{\max }(s)=d$, the probability that the opposite inequality $n_{0} \bar{D}_{k}(d)<D_{k}(s)$ holds tends to zero when $n_{0}$ approaches infinity.

${ }^{3}$ Let $s=\left\{\left(x_{i_{1}}, y_{i_{1}}\right), \ldots,\left(x_{i_{n}}, y_{i_{n}}\right)\right\}$ is a subset of $s_{0}$ having $d_{\max }(s)=d$. Its best horizontal line $y(x)=a$ is obtained for $a=(1 / n) \sum_{k=1}^{n} y_{i_{k}}$. If $s$ is not a subset of $s_{d}$, we translate it by amount $a$ and obtain a set $s^{\prime} \equiv\left\{\left(x_{i_{1}}, y_{i_{1}}-a\right), \ldots,\left(x_{i_{n}}, y_{i_{n}}-a\right)\right\}$ contained in $s_{d}$. Its best horizontal line is $y(x)=0, d_{\max }\left(s^{\prime}\right)=d_{\max }(s)$, so that $D_{k}(s)=D_{k}\left(s^{\prime}\right)$, because the least-square sums for subsets $s$ and $s^{\prime}$ are equal as well. As subset $s_{d}$ contains all points from $s_{0}$ inside boundaries $y= \pm d$, and $d_{\max }\left(s_{d}\right)=d$, it follows that $D_{k}\left(s_{d}\right) \geq D_{k}\left(s^{\prime}\right)=D_{k}(s)$.
} 
The condition for the maximum of (5), i.e. $\partial \bar{D}_{k}(d) / \partial d=0$, leads to a scale-independent equation,

$\left(\frac{d_{b}}{\sigma}\right)^{3} \exp \left[-\frac{d_{b}^{2}}{2 \sigma^{2}}\right]-k \int_{0}^{d_{b} / \sigma} t^{2} \exp \left(-t^{2} / 2\right) \mathrm{d} t=0$,

which relates exponent $k$ to the ratio $d_{b} / \sigma$. In other words, if we choose one of them, e.g. $d_{b} / \sigma$, then the other one $(k)$ will follow from (6). Thus, for $d_{b} / \sigma=1$ we find

$k=\frac{\exp \left(-\frac{1}{2}\right)}{\int_{0}^{1} t^{2} \exp \left(-t^{2} / 2\right) \mathrm{d} t} \approx 2.435$.

Alternatively, if we specify the value of exponent $k$, then the value $d_{b}$ for which $\bar{D}_{k}(d)$ attains a maximum follows from Eq. (6). Thus, for $k=2$ we obtain

$d_{b} / \sigma=1.36876$,

implying that the best subset $s_{b}$ consists of all data points between horizontal lines $y=-d_{b}$ and $y=d_{b}$, containing approximately $83 \%$ of normally distributed data points.

The bottom panel of Fig. 2 presents $k$ versus $d_{b} / \sigma$, for a Gaussian distribution (bold line), according to (6). For comparison, analogous curves $k=k\left(d_{b} / \sigma\right)$ are shown for Lorentzian and for double-exponential distribution.

It should be noted that exponent $k$ is not completely free of constraints. The upper limit is strict and amounts to $k<3$. Namely, from Eq. (6), it follows that $k=\left(z^{3} \mathrm{e}^{-z^{2} / 2}\right) / \int_{0}^{z} t^{2} \mathrm{e}^{-t^{2} / 2} \mathrm{~d} t$, where $z \equiv d / \sigma$. For $z \rightarrow 0$, we also have $t \rightarrow 0$, so that $\mathrm{e}^{-t^{2} / 2} \rightarrow$ 1. Therefore, the upper limit is evaluated as $k=z^{3} / \int_{0}^{z} t^{2} \mathrm{~d} t=3$.

The lower limit is not as strict and cannot be deduced from Eq. (6), which holds in the case of pure Gaussian scatter around a $y=0$ value. Apart from defining the width of the best subset, exponent $k$ has a significant impact on the ability of the DLS method to resolve outliers. When outliers actually do occur, details of bulk data distribution become of secondary importance. The following example illustrates the effect of the exponent $k$ on the order-of-magnitude scale. Consider a set $s_{0}$ containing an odd number $n_{0}=2 m+1$ of points, such that $x_{i}=i,\left(i=1, n_{0}\right), y_{1}=y_{3}=\ldots=y_{2 m-1}=\epsilon, y_{2}=y_{4}=$ $\ldots=y_{2 m}=-\epsilon$, and $y_{2 m+1}=1$. For small $\epsilon$, the last point is a typical outlier. Let us choose the horizontal lines $y(x)=a$ as model functions. Considering that the best horizontal line level $a_{b}$ is equal to the mean value of $y_{1}, y_{2}, \ldots, y_{n_{0}}$, we find $D_{k}\left(s_{0}\right)=\left(1-1 / n_{0}-\epsilon^{2}+n_{0} \epsilon^{2}\right) /\left(1-1 / n_{0}\right)^{k}$ for the whole data set, and $D_{k}(s)=2 m \epsilon^{2-k}$ for the set $s$, obtained by removing the outlying point from $s_{0}$. For sufficiently small $\epsilon$ and $k<2$, it follows that $D_{k}\left(s_{0}\right)>D_{k}(s)$, which implies that the outlier could not be removed if $k<2$. Therefore, we accept the constraint $k \geq 2$ as the lower limit for the exponent $k$, with the remark that in the case of well-behaved data distributions, with soft outliers, DLS will work even if $k<2$, though its ability to resolve outliers will be reduced.

\subsection{Indefinite case}

By removing layer-by-layer from the initial data set, the successive data subsets contain a smaller and smaller number of points. It may happen, eventually, that we have to deal with a subset $s$, such that each of its $n$ data points lies on the corresponding OLS best-fit curve $y=y_{s}(x)$. Then, both $\sum_{s} d_{i}^{2}=0$ and $d_{\max }(s)=0$, so that the density of least squares $D_{k}(s)=$ $\left(\sum_{s} d_{i}^{2}\right) / d_{\max }^{k}(s)$ apparently becomes indefinite.
This difficulty can be resolved by taking into account that the measured data are subject to a limited resolution of measurement. Therefore, although all data points lie on the best-fit curve at the current resolution, it is more likely that their $a c$ tual values are scattered around it according to some "unknown" sub-distribution that is beyond measurement resolution. In other words, a quantity $d_{\max }>0$, assigned to this sub-distribution exists, though not accessible by the measurement technique, characterizes the data set. That being the case, one can rewrite the expression for $D_{k}(s)$ in the form

$D_{k}(s)=\frac{\sum_{i=1}^{n-1} d_{i}^{2}+d_{\max }^{2}}{d_{\max }^{k}}=d_{\max }^{2-k}\left[1+\sum_{i=1}^{n-1} d_{i}^{2} / d_{\max }^{2}\right]$,

where $d_{i}(i=1,2, \ldots, n)$ are actual distances, inherent in the unknown sub-distribution ${ }^{4}$. Hence, it is convenient to introduce the quotient $q$ of the mean-squared distance $\frac{1}{n-1} \sum_{i=1}^{n-1} d_{i}^{2}$ and $d_{\max }^{2}$, i.e.,

$q \equiv \frac{\frac{1}{n-1} \sum_{i=1}^{n-1} d_{i}^{2}}{d_{\max }^{2}}$

as a characteristic parameter of the assumed sub-distribution, such that $D_{k}(s)$ takes the form

$D_{k}(s)=d_{\max }^{2-k}[1+(n-1) q]$.

According to (10), the density of least squares $D_{k}(s)$ is determined by the parameters: $d_{\max }$ and $q(0<q<1)$. Since not accessible to measurements, one may treat both parameters as subject to choice. The exception arises for $k=2$, when (10) simplifies to

$D_{2}(s)=1+(n-1) q$,

with $q$ as a single parameter required to specify $D_{2}(s)$.

One can try to elaborate the choice of $q$ by arguing that any distribution, taken in narrow limits of stochastic variable, should be nearly uniform. Consequently, in the narrow limits set by the resolution of measurement, the presently assumed subdistribution should be uniform as well. Therefore, the expected value of quantity $q$, given by Eq. (9), is

$\langle q\rangle=\frac{\left\langle d^{2}\right\rangle}{d_{\max }^{2}}=\frac{1}{d_{\max }^{2}} \int_{0}^{d_{\max }} t^{2} g(t) \mathrm{d} t$,

which evaluates to $\langle q\rangle=1 / 3$ in the case of uniform distribution $g(t) \mathrm{d} t=\mathrm{d} t / d_{\max }$. Hence,

$q=\frac{1}{3}$

is an appropriate choice according to our experience. In this case, the density of least squares, Eq. (11), amounts to

$D_{2}(s)=1+\frac{n-1}{3}$.

However, if $k>2$ is adopted, an independent estimate of $d_{\max }$ is to be supplied to evaluate $D_{k}(s)$, see Eq. (10). A reasonable choice is to assume that $d_{\max }$ has the same order of magnitude as $d_{r}$, the original resolution of measurement.

\footnotetext{
${ }^{4}$ For simplicity, here we take $d_{\max }=d_{n}$ and $d_{i}<d_{\max }$ for all $i<n$.
} 
If the data set is accompanied by measurement errors $\sigma_{i}$, then the density of weighted least squares is given by Eq. (3). Technically, the foregoing analysis holds, but when applied to relative distances $\delta_{i}=d_{i} / \sigma_{i}$, rather than to geometrical distances $d_{i}$. The density of weighted least squares in the indefinite case is given analogously to Eq. (11) by

$D_{w, k}(s)=\delta_{\max }^{2-k}[1+(n-1) q]$,

which yields $D_{w, 2}(s)=1+\frac{n-1}{3}$, for $k=2$ and $q=1 / 3$. If $k \neq 2$ we also need an independent estimate of measurement resolution, $d_{r}$, to evaluate $\delta_{\max }=d_{r} / \sigma_{\min }$. Here $\sigma_{\min }$ denotes the minimal error pertaining to the subset of points that lie on the weighted least-square best-fit curve.

\section{Error estimation in the best-fit parameters}

We propose a procedure for rough estimation of the uncertainties of DLS best-fit parameters based on parameter $d_{b}$ - the width of DLS best subset. The benefit is most evident when the data set is not accompanied by measurement errors. For the sake of simplicity, we assume that the DLS exponent $k$ is set to 2 , therefore $d_{b} / \sigma=1.36876$.

(i) Measurement errors are not available in the original data set. After running DLS, we assign $\sigma=d_{b} / 1.36876$ to all close points and use these estimated errors in a WLS fitting algorithm. The reported uncertainties we accept as the errors of the DLS best-fit parameters. For data sets containing a small number of points, it may happen that all close points lie on the OLS best-fit curve; i.e. we have to deal with the indefinite case. Since experimental errors are not given, there is no way to estimate uncertainties of the best-fit parameters even for $k=2$, unless the resolution of measurement $d_{r}$ is known. In this case, one can accept $\sigma \sim d_{r}$ as a surrogate for missing experimental variances and proceed with WLS to estimate uncertainties of the best-fit parameters.

(ii) Measurement errors $\sigma_{i}$ are available in the original data set. Instead of $d_{b}$, DLS will give $\delta_{b}$ which is related to the standard deviation $\sigma_{0}$ of the relative residuals $\delta_{i}=d_{i} / \sigma_{i}-$ therefore: $\delta_{b} / \sigma_{0}=1.36876$. Note that, if the individual errors $\sigma_{i}$ are correctly estimated, then $\sigma_{0} \approx 1$ or $\delta_{b} \approx 1.37$. If so, after running the WLS algorithm on the set of close points (with original $\sigma_{i}$ ), the returned uncertainties are accepted. However, if $\sigma_{0}$ is notably different from 1 , it signifies that original measurement errors were badly estimated. In this case, the best one can do is to go back to the experiment, if possible. If not, it is safer to correct original errors than to proceed with wrong ones, especially if $\sigma_{0}>1$. The correction is quite simple: $\bar{\sigma}_{i}=\sigma_{i} \cdot \sigma_{0}$. If we run the WLS algorithm on the set of close points with corrected errors $\bar{\sigma}_{i}$, the reported uncertainties will suit the data scatter. Finally, if the indefinite case occurs while processing a data set with known $\sigma_{i}$, it is enough to apply WLS fit on the subset of close points retaining original errors.

\section{Examples}

For the sake of simplicity, in all our examples we have taken $k=2$ and assumed that no experimental errors have been assigned to data points. The uncertainties are estimated from the data scattering, as discussed above.

In Fig. 3, we present a data set consisting of 11 moderately scattered points. By applying the DLS method to the family of straight-line model functions, we found 3 outlying points,

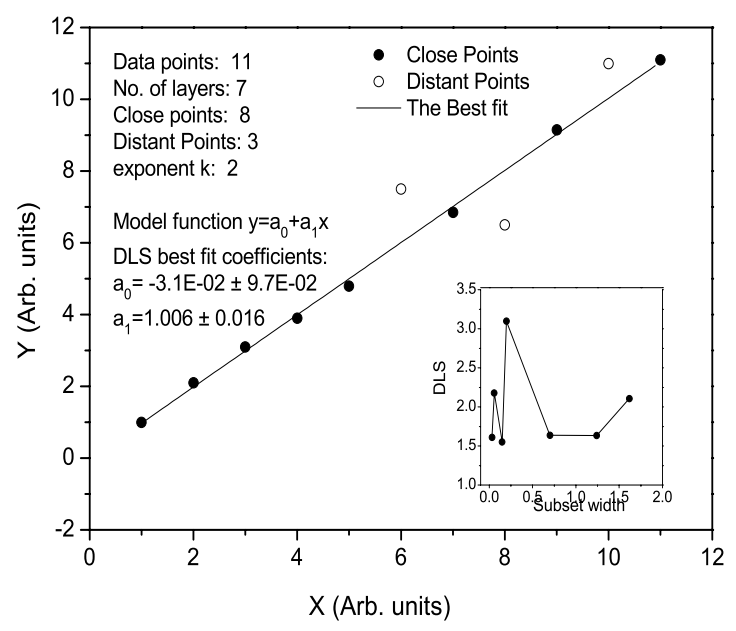

Fig. 3. Eleven moderately scattered points and the best-fit straight line according to the DLS method. Three points are recognized as distant points, i.e. outliers. The remaining eight points are close points. The best-fit parameters are only obtained on the subset of close points only. The inset displays the density of least squares, DLS, versus subset width. The maximum of DLS is clearly noticeable. The density of least squares for the whole data set is shown by the rightmost point. The density of least squares of the subset obtained after the first layer has been removed is presented by the next point and so on. We removed 6 layers, each containing 1 point. The width of the last set analyzed, containing $n=5$ points, is 0.03106 .

while 8 points were recognized as close points. In order to find the best-fit parameters and the corresponding uncertainties, one can simply run WLS for the close points only. In the inset, we present the density of least squares versus width of the consecutive data subsets. The rightmost point represents the density of least squares for the whole data set. The next point represents the density of least squares after the first layer is removed, and so on. The density of least squares attains its maximum after removal of three layers, each containing one point. Therefore, the best subset contains 8 points, recognized as close points.

An artificially generated spectrum with three spectral lines is shown in Fig. 4. The magnitude of applied Gaussian noise is $\sigma=0.04$. To demonstrate stability and robustness of the DLS method, we fitted the horizontal base line with the fourthorder polynomials. Note the pronounced stability of DLS bestfit: though determined by five coefficients, the best-fit polynomial has the appearance of a horizontal line. For the sake of comparison, we also present the best-fit polynomial according to the OLS method and the best-fit polynomial obtained by the robust approach with a Lorentzian probability distribution. The difference is self-evident. The width of the best subset $d_{b}=0.038$ is quite close to the magnitude of the applied Gaussian noise, and it can serve as a distinguishing parameter: points that satisfy $d_{i}>d_{b}$ belong to spectral lines and are treated as formal outliers, while data points satisfying $d_{i}<d_{b}$ are associated to the feature of interest, the base line in our example. The scattering of the data points $d_{i}<d_{b}$ around the best-fit curve can be attributed to noise.

In the next example we compare two methods of data fitting: our DLS method and the multi-component fit (MCF). Here we estimate only the parameters of the He II $468.6 \mathrm{~nm}$ Pashen alpha line $\left(P_{\alpha}\right)$, recorded in a high-voltage pulsed discharge (Djeniže et al. 2004) - see Fig. 5. In the captured spectrum, the central broad line is the $P_{\alpha}$ line, while the two unambiguous peaks, left and right of the central line, correspond to the 


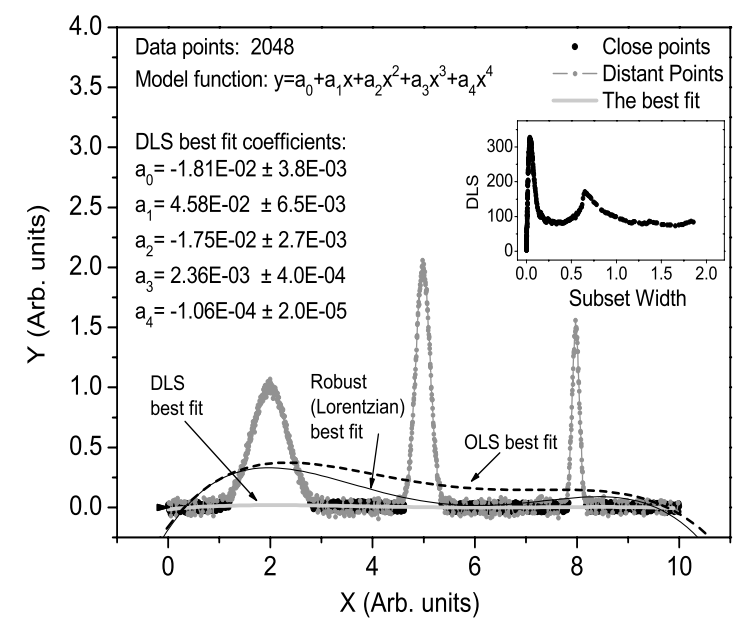

Fig. 4. Artificially generated spectrum with three spectral lines superimposed on line $y(x)=0$. The magnitude of the applied Gaussian noise is $\sigma=0.04$. Fourth-order polynomials are employed as baseline model functions. The solid gray line represents the DLS best-fit polynomial, with respective parameters given in the legend. The dashed line represents the OLS best-fit polynomial, while the solid black line represents the best-fit polynomial obtained by the robust approach with a Lorentzian probability distribution. The inset shows the density of least squares, DLS, versus subset width. The width of the best subset is $d_{b}=0.038$, which is almost the same as the magnitude of the applied noise. In DLS method, $d_{b}$ determines the discrimination level; accordingly, spectral lines are recognized as (formal) outliers.

O II $467.66 \mathrm{~nm}$ and the O II $469.93 \mathrm{~nm}$ lines, respectively. Several low-magnitude peak-like crests visible on both wings are treated as stochastic fluctuations. Note also the presence of two typical outlying points, encircled in the lower panel, probably caused by occasional misfire of the high-voltage trigger. In this particular experiment, the high electron density of the discharge, induces a notable Stark effect. Therefore, the adequate model function for the spectral line is considered to be of the Lorentz profile type $L(\lambda)=h /\left[1+\left(\frac{\lambda-c}{w}\right)^{2}\right]$, depending on wavelength $\lambda$, and on the line parameters: height $h$, center $c$, and half-width $w$. Since the wavelength interval of the spectrum is rather narrow, it is enough to take the base line in the form of a straight line: $y_{b}(\lambda)=a+b \lambda$.

In this problem, the proper DLS model function only consists of the base line and the superimposed He II $P_{\alpha}$ Lorentzian, i.e. $y=y_{b}(\lambda)+L_{\mathrm{HeII}}(\lambda)$. In contrast, if we apply the multicomponent fit, two additional Lorentzians, corresponding to the lateral O II lines, have to be included as well; hence, the proper MCF model function is: $y=y_{b}(\lambda)+L_{\mathrm{HeII}}(\lambda)+L_{\mathrm{OII}}^{(1)}(\lambda)+L_{\mathrm{OII}}^{(2)}(\lambda)$. One can notice that sets of $P_{\alpha}$-line parameters, provided by the DLS fit and by MCF, coincide within the limits of their uncertainties. Note also that, though the base line was an auxiliary feature (not shown in Fig. 5), both methods give a fair agreement between the baseline parameters: the $y$-intercept $a$ and slope $b$.

The specifics of both methods are clearly outlined by the performed parallel fittings. The multi-component fit necessitates a comprehensive form of model function with all (known) features taken into account. In contrast, only the features of interest are incorporated into the DLS model function, as the DLS merit function enables removal of distant points. Some of these points belong to the spectral lines, some are typical outliers, while the rest of them cannot be easy classified. However, when a large number of undocumented features and true outliers are expected, instead of the foregoing straightforward MCF, the use of a

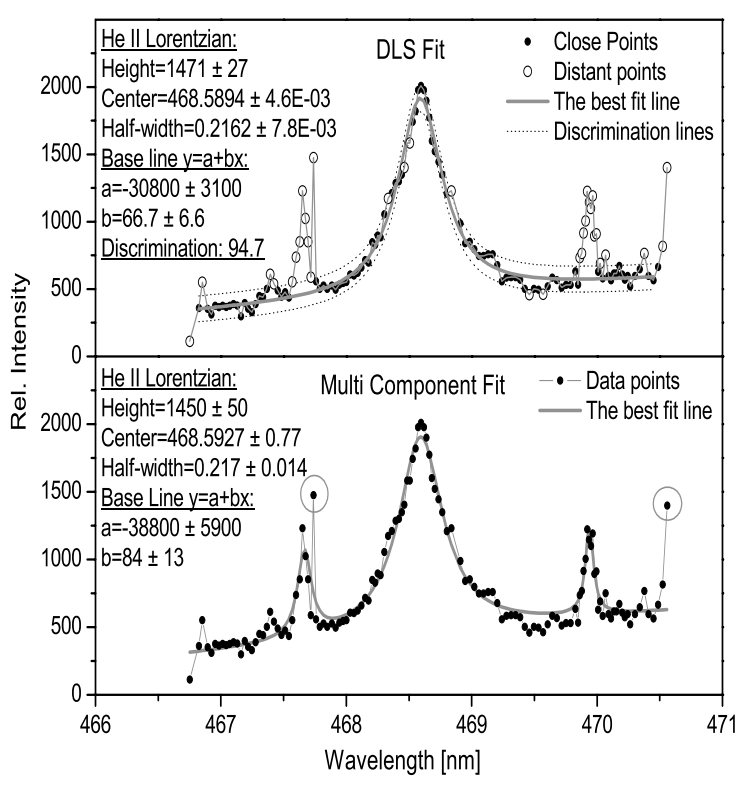

Fig. 5. Comparison of DLS and multi-component fit (MCF) of a spectrum from a high-voltage pulsed discharge. The central line is He II $468.6 \mathrm{~nm}$ Pashen alpha line $\left(P_{\alpha}\right)$. The two lateral peaks are the O II $467.66 \mathrm{~nm}$ and O II $469.93 \mathrm{~nm}$ lines. In DLS fit, the model function has the form $y=y_{b}(\lambda)+L_{\mathrm{HeII}}(\lambda)$, with a straight base line $y_{b}(\lambda)$ of slope $b$ and y-intercept $a$ and a superimposed Lorentzian profile $L(\lambda)=h /\left[1+\left(\frac{\lambda-c}{w}\right)^{2}\right]$ of height $h$, center $c$, and half-width $w$, describing the He II $P_{\alpha}$ line. In MCF, we have also superimposed two Lorentzians, corresponding to the O II lines. The obtained DLS and MCF best-fit parameters coincide within the limits of their errors.

multi-component model function within the DLS approach is recommended to reduce the risk of inaccurate parameter estimation.

In Fig. 6 we present an astrophysical spectrum - Far Ultraviolet Spectroscopic Explorer (FUSE) composite spectrum, originally reported in (Scott et al. 2004). The spectrum continuum is supposed to be of a power-law type $F_{v} \propto v^{\alpha}$. The best-fit power-law exponent quoted in (Scott et al. 2004) is $\alpha=-0.56$ with standard deviation 0.11 . We emphasize that the reported estimate is obtained in a fit performed on selected wavelength regions, free of emission lines: 630-750, 800-820, 850-900, 1095-1100, and 1135-1150 ̊. The solid black line in Fig. 6 shows this fit in wavelength scale, with the power law $F_{\lambda} \propto \lambda^{\alpha_{\lambda}}$ and exponent $\alpha_{\lambda}=\alpha+2=1.44$.

Contrary to the procedure adopted in Scott et al. (2004), the DLS fit is performed on the whole spectrum range (630-1155 of the rest-frame wavelengths; the resulting best-fit curve is represented by the solid gray line in Fig. 6 . The DLS best-fit exponent is $\alpha_{\lambda}^{\mathrm{DLS}}=1.260$, while the standard deviation, relying on the inherent noise, is estimated to 0.034 . Without any prior knowledge taken into account, the DLS best-fit value $\alpha_{\lambda}^{\text {DLS }}$ is slightly less than the one quoted originally. It is indicative that the two tiny wavelength intervals $1095 \AA-1100 \AA$ and $1135 \AA-1150 \AA$, (data in black line on the very right of Fig. 6), recognized in (Scott et al. 2004) as regions of spectrum free of emissions lines, have a significant impact on the best-fit value of the power-law exponent. Inclusion of these intervals into the continuum can be justified only if data pertaining to those intervals are reliable and based on a priori knowledge, which obviously has priority over any general procedure. 


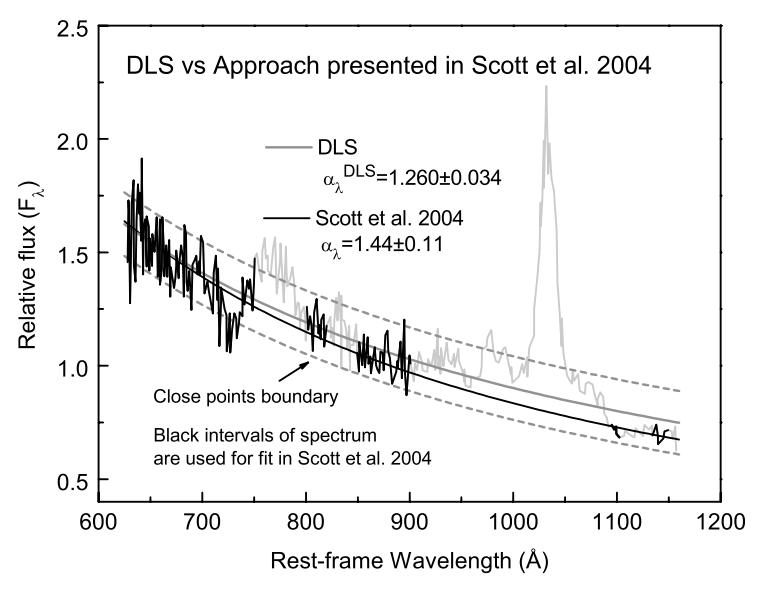

Fig. 6. FUSE composite spectrum originally reported in (Scott et al. 2004). Smooth solid lines represent best-fits of the spectrum continuum to the power-law form $F_{\lambda} \propto \lambda^{\alpha_{\lambda}}$. Scott et al. have applied the fit only to selected wavelength intervals (marked in black) that are free of spectral lines. Their best fit (black line) corresponds to $\alpha_{\lambda}=1.44$. The best power law according to DLS (gray line) is obtained on the whole interval of wavelengths, and it corresponds to $\alpha_{\lambda}^{\mathrm{DLS}}=1.260$. Data for DLS fit are recovered from the original graph with limited accuracy, allowing only a rough comparison of the two approaches.

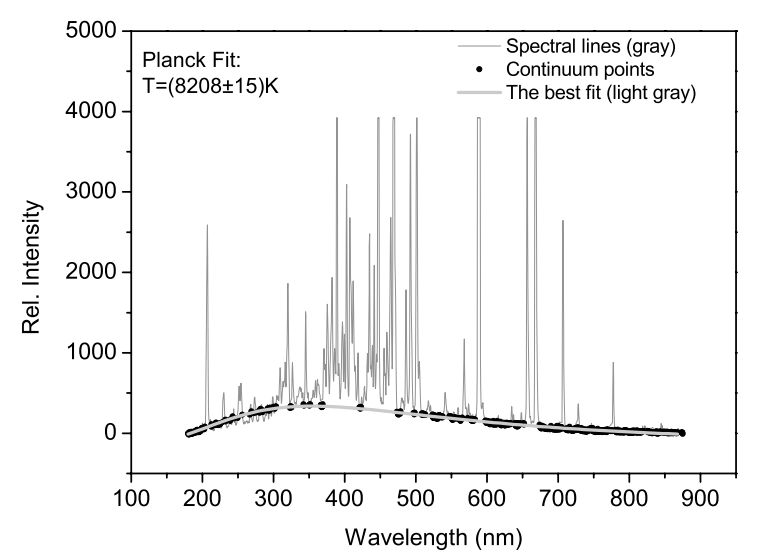

Fig. 7. Time-integrated spectrum of an argon high-voltage pulsed discharge recorded by a pocket-sized Ocean Optics spectrograph in our plasma spectroscopy laboratory. The most intensive spectral lines are clipped, in order to provide a noticeable continuum. By DLS fitting of the spectrum continuum with Planck functions (15), we determined the time-averaged thermodynamic temperature $T=(8208 \pm 15) \mathrm{K}$. Its small uncertainty reflects a low scattering of close points.

However, if we run DLS fit exactly on the same intervals as Scott et al., the trough at about $720 \AA$ is attributed again to distant points signaling that this feature should be considered as a candidate for rejection, even if the corresponding wavelength interval is free of contamination. As mentioned in the preceding example, by applying the DLS method independently, an unbiased review and further insight into the considered problem could be gained.

In Fig. 7 we present a time-averaged spectrum recorded by a miniature Ocean Optics spectrograph during a single pulse of an argon high-voltage pulsed discharge, operating in the Plasma Spectroscopy Laboratory of the Faculty of Physics, University of Belgrade. The spectrum covers the wavelength range from $0.2 \mu \mathrm{m}$ (near UV) to $0.9 \mu \mathrm{m}$ (near IR). The created argon plasma is in a state close to thermodynamic equilibrium (Djeniže et al. 2006), generating a spectrum with a large number of spectral lines and a sizeable continuum. Consequently, the continuum profile defines the base line of the spectrum. The simplest model function follows from the Planck law of black body radiation, which relates the intensity of radiation $I$ at wavelength $\lambda$ with the thermodynamic temperature $T$. Within this example, we employ Planck formula in the form

$I(\lambda ; T)=\frac{c_{1}}{\lambda^{5}} \cdot \frac{1}{\exp \left(\frac{h c}{\lambda k_{B} T}\right)-1}+c_{2}$

where $c_{1}$ comprises all requisite multiplicative factors (including black-body corrections and spectrograph sensitivity), while $c_{2}$ takes the instrumental offset into account. In the present approach, $c_{1}$ and $c_{2}$ are taken as fitting parameters along with $T$. The DLS best-fit Planck function (15) is presented in Fig. 7 by the solid line. The best-fit value of time-averaged thermodynamic temperature is $T \approx 8200 \mathrm{~K}$. An independent temperature estimate based on the Boltzmann-plot technique ${ }^{5}$ gives $T=(8500 \pm 900) \mathrm{K}$. This method requires a large number of pulsed discharge shots and subsequent time averaging, thus adding a significant error to the final result.

\section{Discussion}

In Sect. 2 we defined the merit function (2) on subsets $s$ of a given data set $s_{0}$ as arguments. Since the number of such subsets is typically huge $\left(2^{n_{0}}, n_{0}\right.$ is the number of points in $\left.s_{0}\right)$, it is evident that the search for the merit function minimum by direct inspection would be extremely inefficient. Therefore, some substitute for the standard gradient methods, used in minimization of ordinary functions (e.g. the steepest descent method), has to be proposed.

Suppose that a data set $s_{0}$, comprised of $n_{0}$ points, is given. In order to mimic a single step of a gradient method, we remove point $p$ from $s_{0}$ so that the subset $s_{0}^{(p)}$, obtained by its removal, has the minimum value of merit function among all $n_{0}$ subsets of $s_{0}$ containing $n_{0}-1$ points. Next, we repeat the foregoing procedure on the subset $s_{0}^{(p)}$ instead of on $s_{0}$, and so on. Though it is the closest equivalent to the standard gradient minimization method, this simple iterative procedure is still inefficient because, without some additional criteria, the total number of subsets to be scanned $\left(\sim n_{0}^{2} / 2\right)$ is still large.

However, the structure of merit function (2) implies that the outer points are the best candidates for removal. Indeed, the greater the distance $\left|y_{p}-f\left(x_{p}, \mathbf{a}(s)\right)\right|$ of a discarded point $p \in s$ from the OLS best-fit curve $y=f(x, \mathbf{a}(s))$, the greater the local chances of proceeding to the merit function minimum. Accordingly, if we continue to remove the outermost points, the overall chances of achieving the minimum of merit function (2) are greatest. Any other choice would reduce the prospects of reaching the minimum. Therefore, the ordered collection $C$, introduced in Sect. 2, is an algorithmically optimal assortment of subsets, which provides an efficient way to find the minimum of merit function (2). As in the case of standard minimization, there is no full guarantee that a minimum found within the ordered collection of subsets $C$ is the absolute one.

The rough estimate of errors in the best-fit parameters, introduced in Sect. 4, is based on the assumption that both - ordinary minimization algorithms employed by DLS, as well the DLS itself - are exactly correct. The full confidence limits on

\footnotetext{
5 The Boltzmann-plot technique is a common method for estimating electron temperatures in plasmas at local thermodynamic equilibrium; for some typical applications in astrophysics see (Popović 2003).
} 
DLS best-fit parameters can be determined by the Monte-Carlo method (Press et al. 2001) applied to the set of close points, using $\sigma$ estimated according to Sect. 4.

An important feature of the DLS method is its use of some OLS algorithm as a basic engine. Consequently, for a very broad class of model functions depending linearly on their parameters (e.g. polynomials), the solution is achieved without initial guess. This is a serious advantage with respect to the original CPC method (Bukvić \& Spasojević 2005), which requires the explicit use of a minimization algorithm for all model functions, except for the family of horizontal lines $y=$ const. Nevertheless, if the model function is a nonlinear function of its parameters, then the explicit use of a minimization algorithm, like the Levenberg-Marquardt algorithm (Press et al. 2001), is necessary.

\section{Conclusion}

In this paper, we have proposed a new form of a merit function, which measures the density of least squares (DLS) for subsets of a given data set. The best-fit parameters are obtained by the ordinary least-square method on a subset having a maximum density of least squares. For a rapid approach to DLS maximum a simple algorithm is put forward. Owing to its extreme robustness to the presence of outlying points, DLS method enables flexible data processing in which only features of interest are modeled. The consequence is a notable reduction of fitting parameters and an increased numerical stability, which are important in analyses of complex real-world spectra.

The efficiency of DLS method has been illustrated by several examples. Particularly persuasive is the low uncertainty estimate of thermodynamic temperature from the continuum radiation of a pulsed discharge.

The DLS method of data fitting allows plenty of extensions, which we intend to study in the future.

Acknowledgements. This work was supported by the Serbian Ministry of Science under projects 141010 and 141014 and by the Slovenian Research Agency, under program P2-0056.

\section{Appendix}

\subsection{Statistical underpinning of the DLS method}

From the statistical point of view, many of the data-fitting methods are local-M estimators ${ }^{6}$. The basic assumption of the local$\mathrm{M}$ approach is that the probability density $P^{\prime}\left(y_{m}, x\right)$, of obtaining a measured value $y_{m}$ at given $x$ only depends on the relative deviation $z(\mathbf{a}) \equiv\left(y_{m}-f(x ; \mathbf{a})\right) / \sigma$ from the model function $f(x ; \mathbf{a})$, so that $P^{\prime}\left(y_{m}, x\right)=P^{\prime}(z(\mathbf{a}))$; here, a stands for the true model function parameters, whereas $\sigma$ is the uncertainty of $y_{m}$.

Assuming further that $y_{i}$ 's are independent stochastic variables, the overall probability density (of obtaining a data set $s_{0}=\left\{\left(x_{i}, y_{i}\right): i=1,2, \ldots, n_{0}\right\}$ in a measurement) is $P(\mathbf{a})=$ $\prod_{i=1}^{n_{0}} P^{\prime}\left(y_{i}, x_{i}\right)=\prod_{i=1}^{n_{0}} P^{\prime}\left(z_{i}(\mathbf{a})\right)$.

In practise, the true model function parameters are not known in advance. In the maximum likelihood estimation, the probability density $P(\mathbf{a})$ is taken as a likelihood of parameters, so that the best-fit parameters are those that maximize $P(\mathbf{a})$. Equivalently, one can minimize the merit function $\mu(\mathbf{a}) \equiv$ $-\ln [P(\mathbf{a})]$, commonly written as $\mu(\mathbf{a}) \equiv \sum_{i=1}^{N} \rho\left(z_{i} ; \mathbf{a}\right)$, where $\rho(z ; \mathbf{a}) \equiv-\ln \left[P^{\prime}(z(\mathbf{a}))\right]$.

6 In this subsection we closely follow the terminology and notations used in Press et al. (2001)
The best known local-M method is the OLS method. Here $\rho(z ; \mathbf{a})=z^{2} / 2$, so that the corresponding probability distribution of relative deviations is the Gaussian distribution, $P^{\prime} \sim$ $\exp \left[-z^{2} / 2\right]$. The consequence is that the OLS method provides the best-fit parameters assuming a Gaussian noise. If, however, the noise conforms to some other distribution, then the OLS method will not be the most suitable; for example, in the case of a double-exponential noise, $P(z) \sim \exp (-|z|)$, it is most appropriate to use a local-M estimator with $\rho(z)=|z|$, i.e. to minimize the sum of absolute deviations. When applied to data contaminated by outliers, this least-absolute-deviation method is more robust than the OLS method because it gives less weight to large deviations.

By assigning less and less weight to suspiciously large deviations, Lorentzian and Tukey's biweight methods provide increasingly robust estimates. Eventually, one can decide to discard suspicious points altogether, like in the least-trimmed-square (LTS) method - see (Rousseeuw 1984). The LTS method is based on the metric trimming local-M estimator

$\rho_{c}(z)= \begin{cases}\frac{1}{2} z^{2}, & |z|<c \\ \frac{1}{2} c^{2}, & |z| \geq c\end{cases}$

where constant $c>0$ sets a boundary between $c$-distant points $(|z| \geq c)$ and $c$-close points $(|z|<c)^{7}$. Furthermore, with $\psi(z) \equiv$ $d \rho(z) / d z$, one has $\psi(z)=z$ for $c$-close points, and $\psi(z)=0$ for $c$-distant points ${ }^{8}$. Hence, once recognized, $c$-distant points do not enter the set of equations for the best-fit parameters determined by

$0=\sum_{i=1}^{n_{0}} \frac{1}{\sigma_{i}} \psi\left(z_{i}\right) \frac{\partial f\left(x_{i} ; \mathbf{a}\right)}{\partial a_{k}} \quad k=1, \ldots, m$.

The best-fit parameters, obtained from (17), are actually the bestfit parameters from the OLS method trimmed to the set of $c$-close points.

In what follows, we relate the proposed DLS to the LTS method. To this end, we first demonstrate some of LTS basic features on a pure Gaussian noise (4) with a known standard deviation $\sigma_{i}=\sigma$, and horizontal lines $y(x)=a$ taken as model functions. It follows that the merit function is a sum of two terms,

$\mu_{c}(a)=\mu_{c}^{\prime}(a)+\mu_{c}^{\prime \prime}(a)$,

the term comprising close-points contribution

$\mu_{c}^{\prime}(a) \sim \int_{a-c}^{a+c}(z-a)^{2} \mathrm{e}^{-z^{2} / 2} \mathrm{~d} z$

and the term comprising distant-points contribution

$\mu_{c}^{\prime \prime}(a) \sim c^{2}\left(\int_{-\infty}^{a-c} \mathrm{e}^{-z^{2} / 2} \mathrm{~d} z+\int_{a+c}^{\infty} \mathrm{e}^{-z^{2} / 2} \mathrm{~d} z\right)$.

7 The corresponding distribution is $P^{\prime}(z)=K \mathrm{e}^{-z^{2} / 2}$ for $|z|<c$, and $P^{\prime}(z)=K \mathrm{e}^{-c^{2} / 2}=$ const. for $|z| \geq c$, where $K$ is the normalization constant. Though the particular value of $K$ has no consequence for the analysis that follows note, however, that $K$ depends on $c$ and on the available interval of $z$, which is finite due to a finite experimental range in which the data were collected.

${ }^{8}$ Note that the form of $\rho_{c}$ is unique, if we want to relate it with the DLS method. Indeed, in the DLS method we treat close points like in the OLS method, whereas distant points are discarded. Therefore, in a related local-M approach, one has $\rho(z)=z^{2} / 2$ for close points and $\psi(z)=0$ for distant points, implying that $\rho(z)=$ const. for distant points. The only continuous $\rho(z)$ satisfying both conditions is (16). 
All three quantities, $\mu_{c}(a), \mu_{c}^{\prime}(a)$, and $\mu_{c}^{\prime \prime}(a)$, are even functions of $a$. Their respective derivatives are:

$$
\begin{aligned}
& \frac{\partial \mu_{c}}{\partial a} \sim 4 \int_{0}^{c} z \mathrm{e}^{-\left(a^{2}+z^{2}\right) / 2} \sinh (a z) \mathrm{d} z, \\
& \frac{\partial \mu_{c}^{\prime}}{\partial a} \sim 2 \int_{0}^{c} z^{2} \mathrm{e}^{-\left(a^{2}+z^{2}\right) / 2} \cosh (a z)[z \tanh (a z)-a] \mathrm{d} z, \\
& \frac{\partial \mu_{c}^{\prime \prime}}{\partial a} \sim 2 c^{2} \mathrm{e}^{-\left(a^{2}+c^{2}\right) / 2} \sinh (a c) .
\end{aligned}
$$

From (21) and (23), it can be shown that (for any $c$ ) both $\mu_{c}(a)$ and $\mu_{c}^{\prime \prime}(a)$ are increasing (even) functions of $a$ for $a>0$, so they both attain their respective absolute minimum at $a=0$. In other words, $y=0$ is the best horizontal line, as expected.

However, the close-points contribution $\mu_{c}^{\prime}(a)$ exhibits a less simple behavior. Thus, for large $c^{\prime}$ s, $\mu_{c}^{\prime}(a)$ has two equal and symmetrically positioned maxima. Between the maxima, there is a single local minimum, located at $a=0$; cf. Fig. 8. For decreasing $c$, i.e. for narrowing the boundary, the minimum shallows and the adjacent maxima flatten approaching each other, and eventually merge at $a=0$ as $c$ attains its critical value $c_{c}$. At the value $c_{c}$, which coincides with the solution of Eq. (6) for $k=2$, the local minimum vanishes ${ }^{9}$.

In the region $c \leq c_{c}$, the function $\mu_{c}^{\prime}(a)$ exhibits a single absolute maximum at $a=0$; cf. Fig. 8. Hence, one can find the best-fit parameters by maximizing the close-points contribution $\mu_{c}^{\prime}(a)$ to the merit function, instead of minimizing the merit function $\mu_{c}(a)$ itself. We will use this fact as a link between the LTS and DLS methods.

Now, let us take $c=d_{b} / \sigma$, where $d_{b}$ is the width of the best subset found by the DLS method for a given $k$ - see Sect. 3.1. It can be recognized that the best subset $s_{b}$, having maximum density of least squares $D(s)$, is actually the set of $c$-close points ${ }^{10}$. Furthermore, the LTS best-fit parameters are the OLS best-fit parameters, obtained on the set of $c$-close points. They coincide with DLS best-fit parameters, obtained by the same method (OLS), and on the same subset ( $s_{b}=$ set of $c$-close points). This indicates that DLS best-fit parameters are in fact the maximum likelihood parameters for a suitably chosen boundary value $c$ separating close and distant points in the LTS method.

At the end of this section, we would like to point out one major difference between two methods, which is relevant, in particular, when neither the width of noise nor the extent to which outliers have contaminated the experimental data are known. In the LTS method, the boundary $c$ perfectly suits tuning the extent of contamination, having the role of some kind of confidence level, but requires the width of noise to be known in advance. Though less obvious, the exponent $k$ within the DLS method serves the same purpose, with an essential advantage that in the

${ }^{9}$ For a given $c$, let $a(c)>0$ be the abscissa of the right-hand side maximum of $\mu_{c}^{\prime}(a)$ and let $a=a(c)$ is the line of right-hand side maxima. Along this line $\partial \mu_{c}^{\prime} / \partial a=0$, hence the first derivative of (22) satisfies $0=c^{2} \mathrm{e}^{-c^{2} / 2}[a \cosh (a c)-x \sinh (a c)]+\frac{\mathrm{d} a}{\mathrm{~d} c} \int_{0}^{c} z^{2} \mathrm{e}^{-z^{2} / 2}[(1-$ $\left.\left.x^{2}\right) \cosh (a z)+a \sinh (a z)\right] \mathrm{d} z$. As $a(c) \rightarrow 0$ when $c \rightarrow c_{c}$, the previous equation simplifies to $0=\int_{0}^{c} z^{2} \mathrm{e}^{-z^{2} / 2}\left[1-z^{2}\right] \mathrm{d} z$. Finally, taking into account $\int_{0}^{c} z^{4} \mathrm{e}^{-z^{2} / 2} \mathrm{~d} z=3 \int_{0}^{z} z^{2} \mathrm{e}^{-z^{2} / 2} \mathrm{~d} z-c^{3} \mathrm{e}^{-c^{2} / 2}$, we retrieve (6) for $k=2$. ${ }_{10} \mathrm{As}$ in the DLS method we adopt $2 \leq k<3$, and $c=d_{b} / \sigma$ is obtained from (6), it follows that $c \leq c_{c}$.

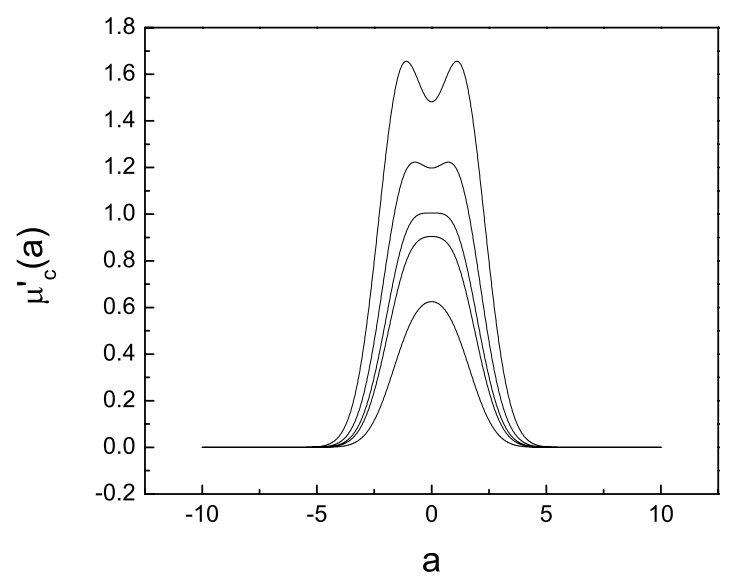

Fig. 8. LTS method: close-points contribution $\mu_{c}^{\prime}(a)$ to merit function, versus horizontal line level $a$ for Gaussian distribution of deviations. The five presented curves in descending order correspond to the boundary $c$ values $1.7,1.5,1.36876,1.3$, and 1.1 , respectively. The critical value $c_{c}=1.36876$, separates the two modes of $\mu_{c}^{\prime}(a)$ behavior. For $c>c_{c}$, the function $\mu_{c}^{\prime}(a)$ exhibits two maxima, separated by a local minimum at $a=0$; when approaching the critical value $c_{c}$ from above, the two maxima merge with the local minimum. For $c \leq c_{c}$, the function $\mu_{c}^{\prime}(a)$ has an absolute and single maximum at $a=0$.

DLS method we do not need to know the width of noise in advance. Instead, the width is estimated by the DLS method itself, together with best-fit parameters.

\subsection{Subroutine DLSFIT}

In this section we present a subroutine DLSFIT that implements the DLS method. The subroutine DLSFIT is not selfcontained, because it needs a linear and a nonlinear weighted least-square (WLS) subroutine to be supplied as working engines. For this purpose, we have chosen well-documented (Press et al. 2001) subroutines: lfit (for general-linear WLS data fitting) and mrqmin (for nonlinear WLS data fitting). These routines, together with the subroutines: zbrent, gaussj, covsrt, and mrqcof (called by DLSFIT, lfit and mrqmin), are available on-line on the official Numerical Recipes (NR) site http://www.nr.com, governed by Cambridge University Press.

Here we present a FORTRAN code of DLSFIT subroutine. To keep it as short as possible, we use concise FORTRAN90 syntax. For the same reason we refer to the simply portable FORTRAN77 (version 2.10) code of subroutines zbrent, gaussj, covsrt, and mrqcof - see, for instance, http://wwW.nrbook. com/a/bookfpdf/f15-7.pdf. The program which calls DLSFIT should be compiled in FORTRAN90 compiler environment with DOUBLE PRECISION as default real kind.

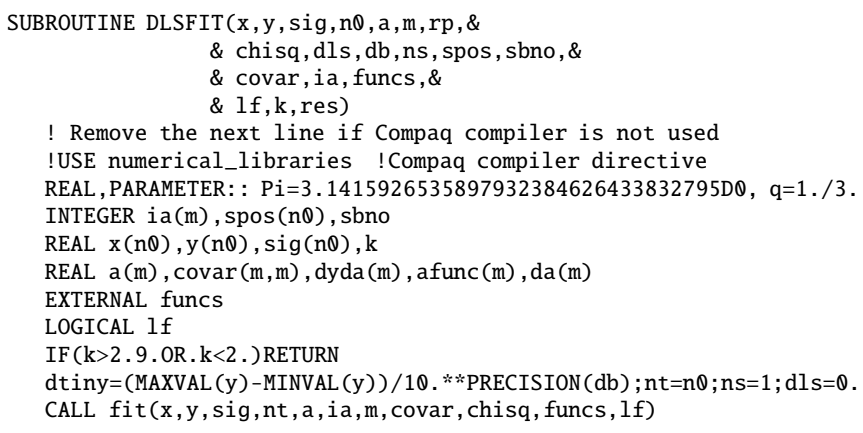


DO WHILE (nt>m+3)

CALL NewMeritVals; IF $(\mathrm{dbt}==0)$ EXIT

END DO

CALL fit ( $x, y$, sig, spos(sbno), a, ia, m, covar, chisq, funcs, lf)

CONTAINS

SUBROUTINE ArrangeLayer

! Rearranges arrays $\mathrm{x}, \mathrm{y}, \mathrm{sig}$ so that their first spos(i)

elements belong to the $i$-th subset in ordered collection $\mathrm{dr}=\mathrm{rp} * \mathrm{dbt} ; \mathrm{ns}=\mathrm{ns}+1$

DO

$\mathrm{i}=1 ; \mathrm{nto}=\mathrm{nt}$

DO WHILE ( $i<=n t)$ ! Remove sublayer

$\mathrm{yc}=\operatorname{fun}(\mathrm{x}(\mathrm{i}))$

$\operatorname{IF}(\operatorname{ABS}(y(i)-y c) / \operatorname{sig}(i)>=d r)$ THEN

$\mathrm{yc}=\mathrm{fun}(\mathrm{x}(\mathrm{nt}))$

IF $(\operatorname{ABS}(\mathrm{y}(\mathrm{nt})-\mathrm{yc}) / \operatorname{sig}(\mathrm{i})<\mathrm{dr})$ THEN

swap $=x(i) ; x(i)=x(n t) ; x(n t)=$ swap

swap=y (i) ; $(i)=y(n t) ; y(n t)=s w a p$

$\operatorname{swap}=\operatorname{sig}(i) ; \operatorname{sig}(i)=\operatorname{sig}(n t) ; \operatorname{sig}(n t)=\operatorname{swap}$ END IF

ELSE

$i=i+1$

END IF

END DO

$\operatorname{IF}((n t==n t o) .0 R .(n t<=m+3)) \operatorname{EXIT}$ END DO

END SUBROUTINE ArrangeLayer

SUBROUTINE NewMeritVals

! Calculates new DLS value

$\operatorname{spos}(\mathrm{ns})=\mathrm{nt} ; \mathrm{dbt}=0$

DO $i=1, n t$

$d=\operatorname{ABS}(y(i)-\operatorname{fun}(x(i))) / \operatorname{sig}(i) ; \operatorname{IF}(d b t<d) d b t=d$

END DO

IF (dbt $>$ dtiny) THEN

$\mathrm{dlst}=\mathrm{chisq} /(\mathrm{dbt} * \mathrm{k})$

ELSE

os $2=\operatorname{SuM}((/(1 . /(\operatorname{sig}(i) * \operatorname{sig}(i)), i=1, n t) /)) / n t$

dlst $=02^{*}\left(1 .+q^{*}(n t-1)\right) * r^{*} *(2-k) ; d b t=0$

END IF

IF (dlst $>$ dls $)$ THEN

$\mathrm{dl} s=\mathrm{dl} \mathrm{st} ; \mathrm{sbno}=\mathrm{ns} ; \mathrm{db}=\mathrm{dbt}$

END IF

END SUBROUTINE NewMeritVals

FUNCTION fun( $\mathrm{x}$ ) ! For given $\mathrm{x}$, calculates value of model function IF (lf) THEN

ELSE

$$
\text { CALL funcs }(x, \text { afunc, } m \text { ); fun=DOT_PRODUCT }(a, \text { a func })
$$

END FUNCTION fun

END SUBROUTINE DLSFIT

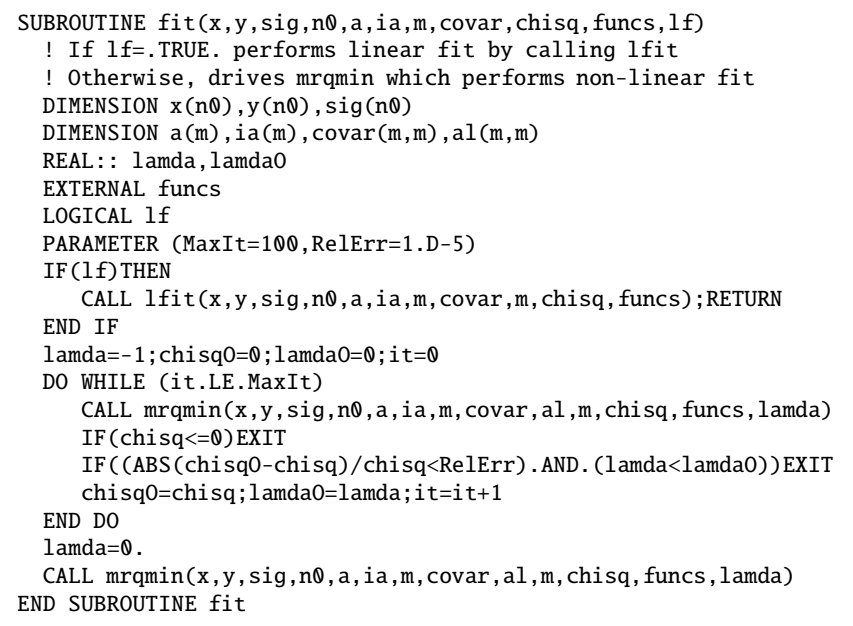

The user supplies to DLSFIT subroutine a set of no experimental data points $\mathrm{x}(1: \mathrm{n} \theta), \mathrm{y}(1: \mathrm{n} \mathbb{\theta})$, with individual standard deviations $\operatorname{sig}(1: n \theta)$ of their $y_{i}$ 's; if $\operatorname{sig}(1: n \theta)$ are not available, then set all sig(i)=1 prior to DLSFIT call. The next argument in the DLSFIT calling list is array $\mathrm{a}(1: \mathrm{m})$ of length $\mathrm{m}$. On input, the user supplies initial values of model function parameters in $\mathrm{a}(1: \mathrm{m})$ in the case when the model function is a nonlinear function of its parameters; otherwise, the input values supplied in $\mathrm{a}(1: \mathrm{m})$ are irrelevant. On output, the main result of DLS method - the best-fit parameters associated with the best subset - are returned in $\mathrm{a}(1: \mathrm{m})$.

The input parameter $\mathrm{rp}$ is the removal parameter, introduced in Sect. 2. In our experience, when $0.9 \leq \mathrm{rp} \leq 1$ the best-fit parameters returned for different values of $\mathrm{rp}$ coincide within the error margins ${ }^{11}$.

Six parameters (chisq, dls, db, ns, spos, sbno), which follow in DLSFIT calling list, are output parameters. Thus, chisq is $\chi^{2}$ and dls is the density of least squares obtained on the best subset in the ordered collection $C$ (see Sect. 2), found by DLSFIT, while $\mathrm{db}$ is the width of the best subset. Additionally, ns is the total number of subsets in $C, \operatorname{spos}(1: n s)$ is an integer array that contains the number of data points in each subset from $C$, and sbno is the ordinal number of the best subset in $C$.

Note that the arrays $x(1: n \theta), y(1: n \theta)$ and $\operatorname{sig}(1: n \theta)$ are reordered on output so that the first $\operatorname{spos}(i)$ data points belong to the subset $s_{i} \in C$. In this way, the user can obtain relevant data for each subset $s_{i} \in C$ by applying the OLS method on the first $\operatorname{spos}(i)$ elements of arrays $x, y$, and sig.

The next three parameters (covar, ia, funcs) in the calling list of the DLSFIT subroutine are specific to the applied lfit and mrqmin NR subroutines. Thus, $\operatorname{covar}(1: \mathrm{m}, 1: \mathrm{m})$ is a twodimensional array, which on output contains the elements of covariance matrix. The integer array ia $(1: \mathrm{m})$ provides a convenient way to keep some of the parameters frozen on their input values $(i a(j)=0$ for frozen $a(j)$, otherwise $i a(j)$ is non-zero); the values of all frozen parameters must be supplied on input in array a. Finally, funcs is the name of a usersupplied subroutine (fpoly from Press et al. 2001 in the case of polynomial fit) proceeded to DLSFIT when DLSFIT is called; when a general-linear fit is performed, then funcs ( $x$, afunc,$m$ ) should return the $m$ basis functions evaluated at $x$ in the array afunc $(1: m)$. In the opposite case, funcs is a user supplied subroutine having syntax funcs $(\mathrm{x}, \mathrm{a}, \mathrm{y}, \mathrm{dyda}, \mathrm{m})$. For given $\mathrm{x}$ and model function parameters $\mathbf{a}$, supplied in array $\mathrm{a}(1: \mathrm{m})$ of length $\mathrm{m}$, it returns the calculated value $f(x ; \mathbf{a})$ of model function in variable $\mathrm{y}$, together with values of all model function partial derivatives $\frac{\partial f}{\partial a_{i}}(x ; \mathbf{a})$, returned in array $\operatorname{dyda}(1: \mathrm{m})$.

The last three parameters in DLSFIT calling list are $l f, k$, and res. The first of them, $1 f$, is a LOGICAL input parameter. When $l f$ is set to . TRUE ., then DLSFIT performs general-linear WLS data fitting by calling lfit subroutine; otherwise, nonlinear fit is performed with an aid of mrqmin subroutine. The value of DLS exponent $k$ is supplied to DLSFIT subroutine in the second parameter of the group, $\mathrm{k}$. Finally, we must also supply the value of $d_{\max }$ (or $\delta_{\max }$ ) used in DLS calculations in the indefinite case - see Sect. 3.2. This is supplied through parameter res the original resolution of measurement.

Note that the DLSFIT does not return errors in the best-fit parameters. Estimation of errors, detailed in 4, is left to the user as it depends on the specifics of actual data; nonetheless, a fullcode example is given in the online material only available in electronic form at the CDS.

At the end, we explain the subroutine fit, called by DLSFIT routine. The calling parameters have the same meaning as for the DLSFIT routine. If model function depends linearly on its parameters, then the subroutine fit calls NR routine $1 \mathrm{fit}$, which performs the general linear WLS data fitting. Otherwise, it serves

\footnotetext{
11 Note that the bigger the value of the removal parameter, the bigger the number of subsets found by DLSFIT, and the longer the execution time, which is of particular importance in the case of large data sets.
} 
as a simple driver for the NR routine mrqmin, used for a nonlinear WLS data fitting. The subroutine fit is singled out from DLSFIT in order to provide a way for flexible estimation of the best-fit parameters on any subset from the ordered collection of subsets $C$ obtained by DLS method.

\section{References}

Barnett, V., \& Lewis, T. 1994, Outliers in statistical data, 3rd edn. (New York: John Wiley and sons)

Bukvić, S., \& Spasojević, Dj. 2005, Spectrochim. Acta, Part B, 60, 1308

Contini, M., \& Viegas, S. M. 2000, ApJ, 535, 721

Dietrich, M., Crenshaw, D. M., \& Kraemer, S. B. 2005, ApJ, 623, 700

Dietrich, J. P., Clowe, D. I., \& Soucail, G. 2002, A\&A, 394, 395

Djeniže, S., \& Bukvić, S. 2001, A\&A, 365, 252

Djeniže, S., Bukvić, S., Srećković, A., \& Platiša, M. 2004, A\&A, 424, 561

Djeniže, S., Srećković, A., Bukvić, S., \& Vitas, N. 2006, Z. Naturforsch. A, submitted

Freudling, W., Corbin, M. R., \& Korista, K. T. 2003, ApJ, 587, L67

Gabel, J. R., Arav, N., Kaastra, J. S., et al. 2005, ApJ, 623, 85G

Gilbert, K. M., \& Peterson, B. M. 2003, ApJ, 587, 123
Hampel, F. R., Ronchetti, E. M., Rousseeuw, P. J., \& Stahel, W. A. 1986, Robust Statistics, The approach based on influence function (New York: John Wiley and sons)

Hoaglin, D. C., Mosteller, F., \& Tukey, J. W. 1983, Understanding robust and exploratory data analysis (New York: John Wiley and sons)

Liu, Q. Z., \& Yan, J. Z. 2005, New Astr., 11, 130

Liu, R. C., Markatou, M., \& Tsai, C. L. 2005, Int. J. Pure Appl. Math., 21, 525

Marx, B. D. 1996, Technometrics, 38, 374

Meisel, L. V., \& Cote, P. J. 1992, Phys. Rev. B, 46, 10822

Motulsky, H. J., \& Brown, R. E. 2006, BMC Bioinformatics, 7, 123

Peterson, B. M., Berlind, P., Bertram, R., et al. 2002, ApJ, 581, 197

Popopović, L. Č. 2003, ApJ, 599, 140

Press, W. H., Teukolsky, S. A., Vetterling, W. T., \& Flannery, B. P. 2001, Numerical Recipes in Fortran 77: The Art of Scientific Computing, Vol. 1 of Fortran Numerical Recipes, software version 2.10 (Cambridge: Cambridge University Press)

Rossa, J., Dietrich, M., \& Wagner, S. J. 2000, A\&A, 362, 501

Rousseeuw, P. J. 1984, J. Am. Stat. Assoc., 79, 871

Scott, J. E., Kriss, G. A., Brotherton, M., et al. 2004, ApJ, 615, 135

Spasojević, Dj., Bukvić, S., Milošević, S., \& Stanley, E. 1996, Phys. Rev. E, 54, 2531

Wang, J., Wei, J. Y., \& He, X. T. 2005, New Astron., 10, 353 